\title{
Population structure of hypervariable Y-chromosomes in Rumex acetosa
}

\author{
A. S. Wilby and J. S. Parker
}

School of Biological Sciences, Queen Mary College, Mile End Road, London E1 4NS.

Males of the dioecious angiosperm Rumex acetosa have a single metacentric $\mathrm{X}$ and two heterochromatic Y-chromosomes. The Ys are constant in size but hypervariable in morphology with centromeres positioned anywhere within the central 40 per cent of the chromosome. 280 males from 28 populations contained 129 variants determined by feulgen staining alone, and samples of 10 seed-grown males from single populations contain between 4 and 8 variants. Similar levels of variation characterise mature males, with variants occurring once or only a few times in a population. Each population has a distinctive spectrum of $\mathrm{Y}$-variants even when $\mathbf{4 0}$ males are examined, and closely adjacent populations are as dissimilar as distant populations. Small, isolated populations carry as many Y-variants as large, continuous populations although they are homozygous for autosomal polymorphisms. In addition to centric shifts, populations usually have pairs of variants differing only in pairing segment location, suggesting that telomere switching may regularly occur. It is argued that this pattern of variation results from neutrality of the variants coupled with an extremely high rate of repositioning of the primary constriction.

\section{INTRODUCTION}

Rumex acetosa is one of only 13 species of dioecious angiosperms which possess morphologicallydistinguishable sex-chromosomes (Westergaard, 1958). Female (pistillate) plants carry a pair of metacentric X-chromosomes while male (staminate) plants have a single $\mathrm{X}$ and two $\mathrm{Y}$-chromosomes distinguished by length designated $Y_{1}$ and $\mathrm{Y}_{2}$ (Ono, 1935). The $\mathrm{X}$ is constant in morphology but the Ys by contrast are extraordinarily variable in structure. In each $\mathrm{Y}$ the centromere can occur anywhere within the central 40 per cent of the chromosome but is excluded from the two distal 30 per cent regions (Wilby and Parker, 1986). On the basis of centromere location in $\mathrm{Y}_{1}$ and $\mathrm{Y}_{2}$ alone, Wilby \& Parker (1986) were able to distinguish 68 variants in only 270 males of $R$. acetosa.

In this paper the structure of natural populations with respect to the hypervariable Y-chromosomes is considered. A total of 36 British populations have been examined and all have proved to be extremely heterogeneous. The extent of variation is not correlated with population size, geographical location or nature of the habitat. It is suggested that the $\mathrm{Y}$-variation is selectively neutral and is maintained by an extremely rapid rate of change.

\section{MATERIALS AND METHODS}

Rumex acetosa is a wind-pollinated perennial herb, primarily a constituent of permanent grassland, road verges and heathland showing a preference for acid soils. It is very widespread in the holarctic region and in Britain it occurs almost everywhere although populations are thinly-scattered and rather small in the drier, highly arable areas of eastern England.

Mature plants were collected from nine populations in England (fig. 1). Five of these were from a small area of north Cornwall, two were collected on lead mines in Shropshire and Derbyshire, while the remaining two were in permanent grasslands in Essex and Cumbria.

Seed collections were made in 31 populations throughout Britain. In three of these, 40 females were individually sampled, while in the remainder 10 were sampled (fig. 2). One male offspring of each female was examined, giving random samples of 40 and 10 males respectively.

Chromosome analyses were made on roots fixed in $1: 3$ acetic alcohol after 0.05 per cent colchicine treatment for two hours, and on buds fixed in Carnoy and stained with 2 per cent acetocarmine. 


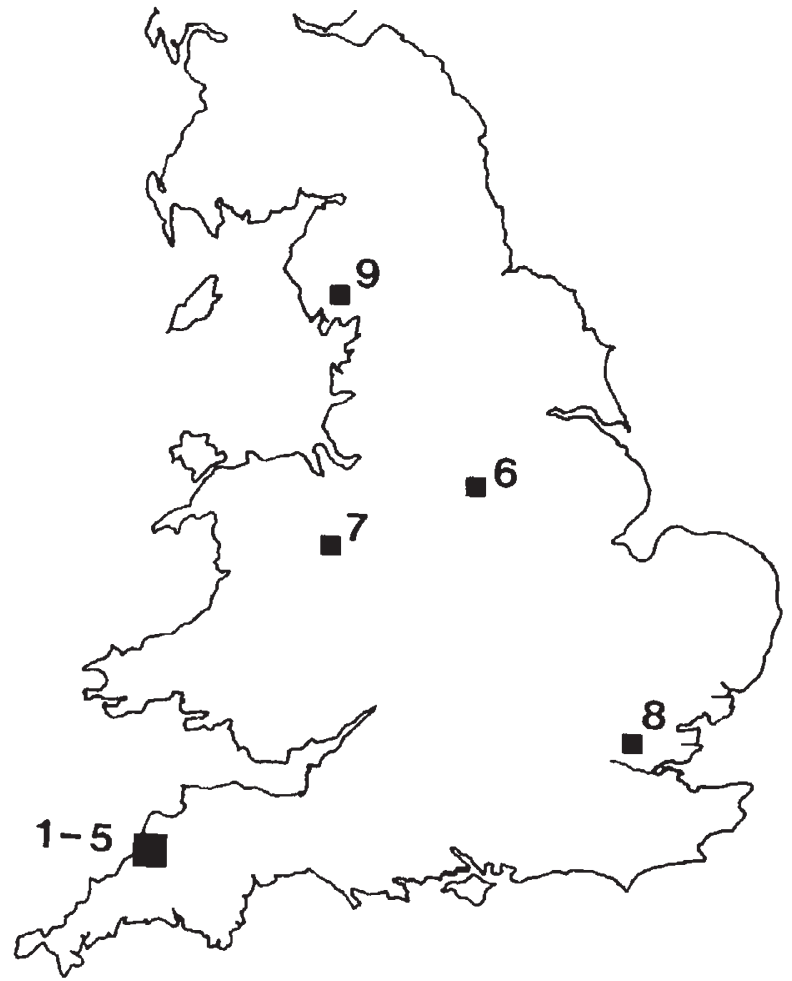

Figure 1 Map showing the 9 English populations from which mature plants of Rumex acetosa were collected. Locations are given in Table 1.

\section{RESULTS}

\section{Chromosome variation in $Y$-chromosomes}

The chromosome complement of female Rumex acetosa is $2 n=12+\mathrm{XX}$. There are six pairs of acrocentric autosomes and a pair of metacentric $\mathrm{X}$ chromosomes about twice the length of the largest autosome. Males have a single $\mathrm{X}$ and two Y-chromosomes, distinguished by size $(2 n=12+$ $X Y_{1} Y_{2}$; fig. 3). The larger $\left(Y_{1}\right)$ is about 85 per cent of the length of the $\mathrm{X}$ at $c$-metaphase while the smaller $\left(\mathrm{Y}_{2}\right)$ is about 75 per cent (Wilby and Parker, 1986). Both Ys are heterochromatic except for a minute telomeric euchromatic segment on each which is homologous to a terminal region on one arm of the $X$. The pairing segments allow us to distinguish the $\mathrm{Y}$-telomeres at mitotic prophase.

The Y-chromosome centromeres are extremely variable in position. Centromeres can be located anywhere within the central 40 per cent of the $Y$ but are never found in the two distal regions. The distribution of centromere location is effectively continuous without preferred sites. Since the two Ys vary independently a huge number of iden-

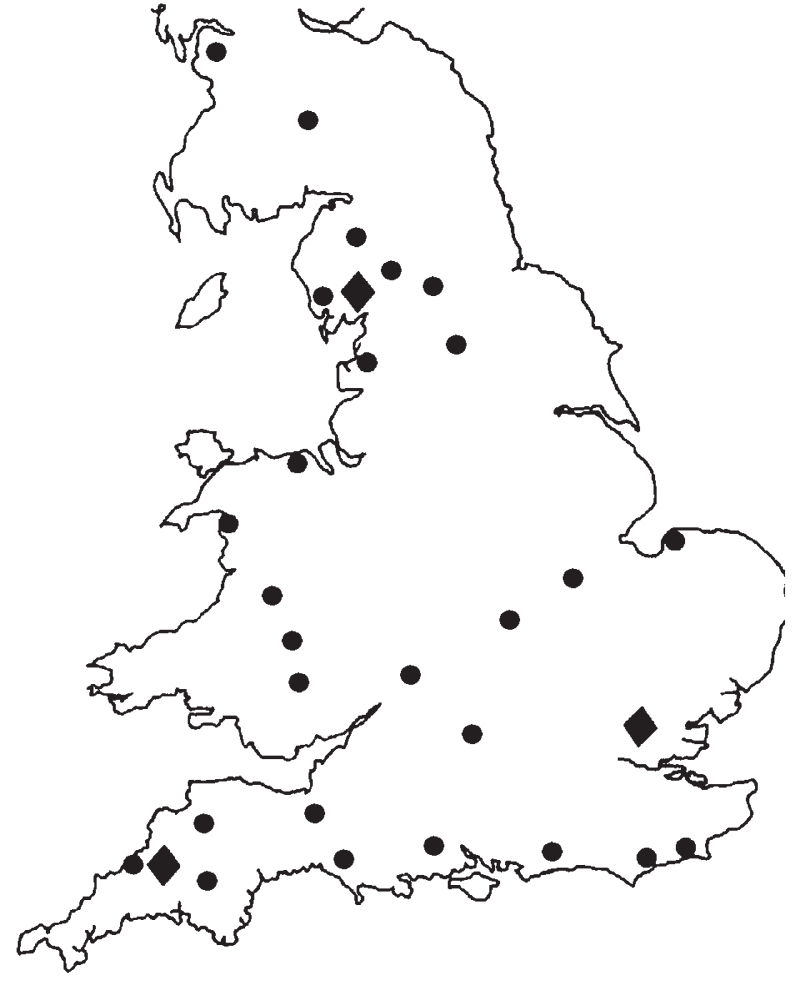

Figure 2 Map showing the 31 British populations from which seed samples were collected. $O=10$-male samples; $\bullet=$ 40-male samples.

tifiable variants can be generated; 68 were found in 270 males in the initial study (Wilby and Parker, 1986). The 390 males considered in this paper can be ascribed to 151 variants.

The terminology that has been employed to refer to different classes of variants is $m$ for a metacentric and $a$ for an acrocentric Y, with the designation " + ' or "- ' depending whether the centromere is closer to or further away from the pairing segment. Thus a plant may have the constitution $\mathrm{ma}^{+}$with a metacentric $\mathrm{Y}_{1}$ and an acrocentric $\mathrm{Y}_{2}$. The asymmetry of acrocentrics is expressed as the long-arm proportion of total length. It is impossible to score polarity reliably in Ys with arm-ratios of less than $0 \cdot 54$.

\section{Population structure of the 10-male seed populations}

\section{$Y$-variant frequency}

A total of 280 males from 28 populations have been examined. Only 85 ( 31 per cent) of these were of Y-constitution $\mathrm{mm}$, with both Ys metacentric. 


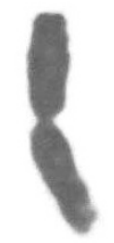

$\mathrm{X}$

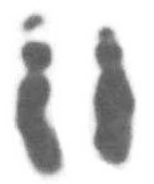

3

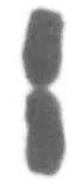

$Y_{1}$

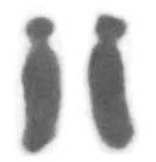

4

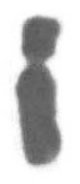

$Y_{2}$

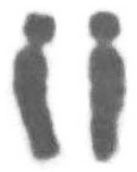

1

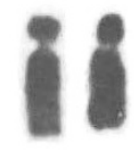

5

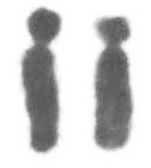

2

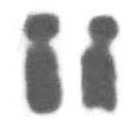

6

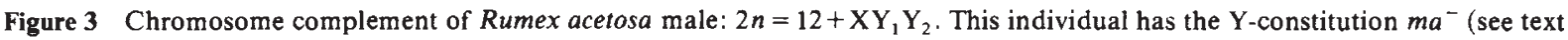
for details of terminology).

Up to six $\mathrm{mm}$ males were found in a sample, and one population contained no males of this type. On average there are $3.1 \mathrm{~mm}$ plants per population. Despite the range of centromere positions covered by the designation $m( \pm 0.03$ for each $Y)$, there is no superabundance of this class. The remaining 70 per cent of males have at least one $\mathrm{Y}$ acrocentric.

There are eight major acrocentric classes (see table 1) and all have been found in the sample, although not with equal frequencies. Indeed the classes $a^{+} a^{-}$and $a^{-} a^{+}$have been found in only 9 individuals.

The most striking feature of population structure is the heterogeneity of the $\mathrm{Y}$-variants. Between 4 and 8 distinct variants were found in the populations, with an average of 5.6 different $\mathrm{Y}$-variants in each ten-male sample (table 1). Thus every second male plant is identifiable. The eight $Y$ variants from the Hawes population are illustrated (fig. 4).

Even this amount of variation will be an underestimate for a variety of reasons. Within the class $\mathrm{mm}$ there will be considerable diversity since this spans 6 per cent difference in arm-ratio for each Y. Also, within single populations acrocentric Ys of the same polarity which differ by less than 3 per cent in arm-ratio have been classified together. Finally, apparently identical Y-variants may have arisen by different evolutionary pathways but finer methods of discrimination are required to establish this.

The Y-chromosome heterogeneity is emphasised by a further consideration. Within single populations, most acrocentric $\mathrm{Y}$-variants are represented only once, and single variants have

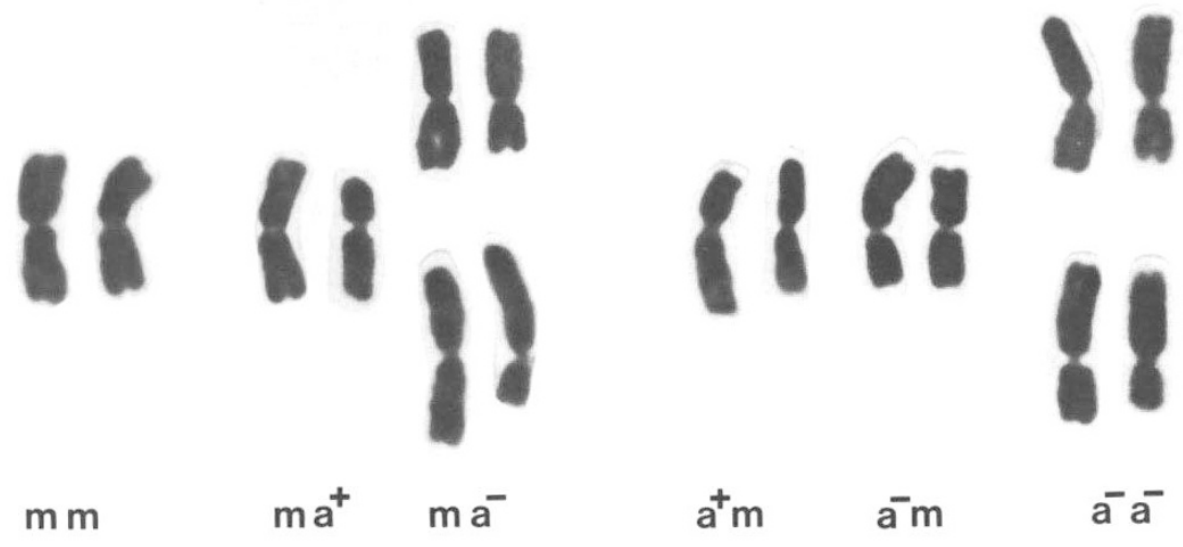

Figure 4 The eight Y-variants found in the 10-male seed samples from Hawes, North Yorkshire. Arm-ratios are given in Table 1. 
Table 1 The types and frequencies of Y-variants found in 28 populations of Rumex acetosa. Ten males were examined in each population. Arm-ratios of acrocentric Ys are given as long-arm length/total length and superscripts represent the frequency of each variant. See text for details of terminology

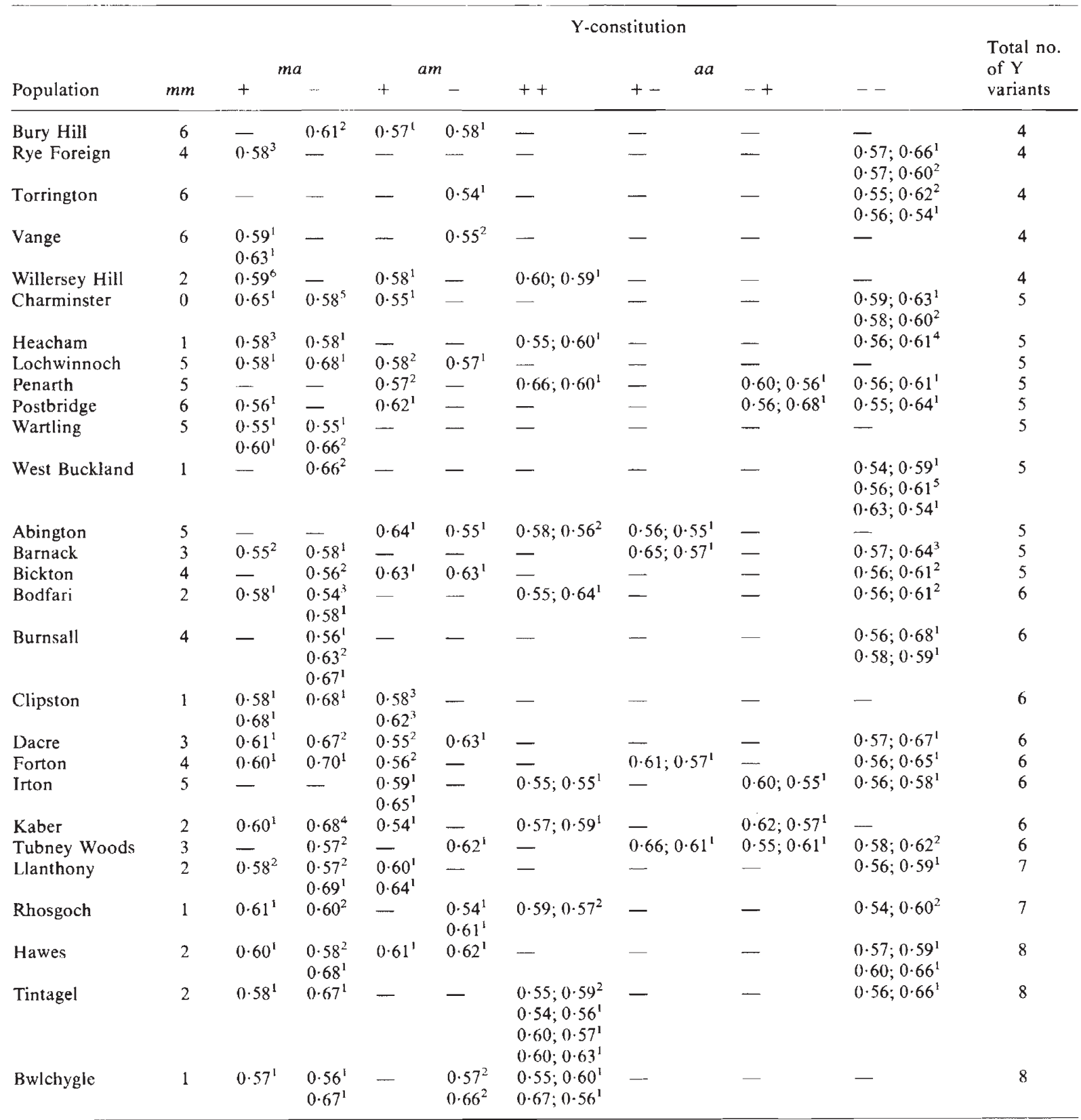

been found more than three times in only 5 of the 28 populations. On average, each variant is represented only 1.45 times. Each population displays a unique pattern of Y-variation (table 1), as if each represents a random sample drawn from an enormous pool of variants.

\section{Relationships of Y-variants, population size and habitat}

The 28 populations were distributed throughout Britain in a wide variety of habitats, including lowland watermeadows, upland heathland, and 
roadside verges in intensely arable regions. These populations also differed greatly in size, those in the west and north being extremely large, while those in the highly arable regions of eastern and southern England are often confined to isolated patches of rough grassland or roadsides supporting very few individuals.

We would expect the smaller populations to be more homogeneous in Y-structure than the enormous, effectively continuous populations of the west and north. The populations can be classified as 13 small and 15 large and in these groups the mean numbers of $\mathrm{Y}$-variants per 10 males are 5.39 and 5.63 respectively, indicating that the variation is largely independent of population size. Now $R$. acetosa in Britain is also variable in the autosomes with three widespread polymorphismsheterochromatic supernumerary segments on autosomes 1 and 6 (fig. 5) and B-chromosomes-and a variety of unique cytological variants such as centric shifts and interchanges. The number of distinct karyotypes, excluding those attributable to Y-variation, has been assessed in 20 individuals per population. This ranges from 2 ( $\delta$ and $q$ ) to 12. Unlike the $Y$-variation, this is related to population size, with 4.60 and 6.67 different karyotypes in small and large populations respectively.

A further method of assessment is the average heterozygosity of each population with respect to the two common autosomal polymorphisms. Again this is related to population size with 0.337 in large populations and only 0.096 in small populations. Neither of these measures is correlated with the number of Y-variants which are apparently indifferent to the pressures of population size.

A plot of Y-variant frequencies reveals no relationships with gross geographical features, no clines or area effects. Also, populations from all habitat types are equally variable. Thus, the Y-

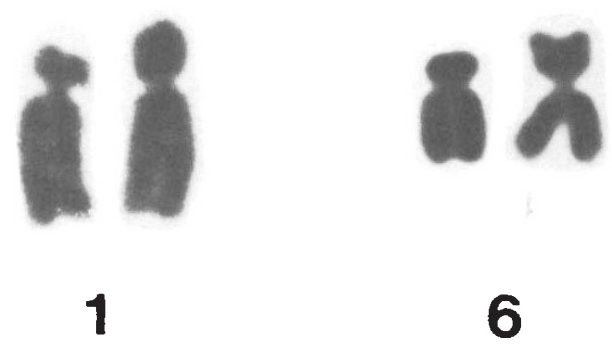

Figure 5 Chromosomes 1 and 6 of Rumex acetosa showing a standard chromosome (left) and a chromosome carrying a heterochromatic supernumerary segment on the short arm (right). Both are polymorphic in natural populations.

variation of $R$. acetosa characterises all populations and, remarkably, is unaffected by both selective and random effects.

\section{Population structure of the 40-male seed populations}

Is the pattern of $Y$-variation the same in all populations such that differences between populations are merely due to sampling error? To test this, 40 males in three populations from contrasting habitats were examined. These were Navestock Heath, Essex (waterlogged ungrazed heath), Torver, Cumbria (permanent sheep-pasture) and Hendra, Cornwall (roadside hedgebank).

Interestingly the number of variants in these populations is only slightly higher than in the 10 -male samples with 11,11 and 7 variants respectively (table 2). As before the class $\mathrm{mm}$ predominates while each acrocentric variant occurs with low frequency. The maximum number of occurrences of an acrocentric is six for an $\mathrm{ma}^{-}$variant at Navestock Heath. The patterns of Y-variation in these three populations remain distinct, with no evidence of a merging of differences.

Table 2 The types and frequencies of Y-variants found in samples of 40 males from each of 3 populations

\begin{tabular}{|c|c|c|c|c|c|c|c|c|c|c|}
\hline \multirow[b]{3}{*}{ Population } & \multirow[b]{3}{*}{$m m$} & \multicolumn{8}{|c|}{ Y-constitution } & \multirow{3}{*}{$\begin{array}{l}\text { Total no. } \\
\text { of Y } \\
\text { variants }\end{array}$} \\
\hline & & \multicolumn{2}{|c|}{$m a$} & \multicolumn{2}{|c|}{$a m$} & \multicolumn{4}{|c|}{$a a$} & \\
\hline & & + & - & + & - & ++ & +- & -+ & -- & \\
\hline Navestock & 16 & $\begin{array}{l}0.54^{1} \\
0.57^{1}\end{array}$ & $\begin{array}{l}0.59^{6} \\
0.63^{3}\end{array}$ & - & $0 \cdot 56^{x}$ & - & $0.60 ; 0.61^{1}$ & - & $\begin{array}{l}0.55 ; 0.60^{4} \\
0.65 ; 0.64^{2} \\
0.62 ; 0.55^{1} \\
0.57 ; 0.61^{2}\end{array}$ & 11 \\
\hline Torver & 23 & $\begin{array}{l}0.57^{2} \\
0.64^{2}\end{array}$ & $0.71^{1}$ & $\begin{array}{l}0 \cdot 55^{1} \\
0 \cdot 61^{3} \\
0 \cdot 66^{1}\end{array}$ & $\begin{array}{l}0.57^{2} \\
0.61^{2}\end{array}$ & $0.53 ; 0.62^{1}$ & - & 一 & $0.55 ; 0.68^{2}$ & 11 \\
\hline
\end{tabular}


Two reasons may account for the relatively small number of variants detected in these populations. Firstly, these populations, perhaps by chance, have a higher average frequency of $\mathrm{mm}$ variants than the smaller samples ( 54 per cent vs. 31 per cent). Secondly, the criterion of 3 per cent difference in arm-ratio between variants was maintained for these large samples. Thus if centromere distribution is essentially continuous (see Wilby and Parker, 1986) with increasing sample size each category will include an ever more heterogeneous group of individuals.

The evidence of the three 40-male samples, emphasises the extreme heterogeneity of the $\mathrm{Y}$ variation system.

\section{$Y$-variation in mature population plants}

The extensive $Y$-variation described above characterises seed-grown males raised under low competition conditions in a greenhouse. It is possible that mature males in natural populations may exhibit a narrower spectrum of variation. To test this, mature males from nine populations were examined. In these, the variation pattern of seedgrown plants was repeated: 36 per cent of males were constitution $\mathrm{mm}$; individual acrocentric variants were detected only once or twice in any sample; all populations had distinctive Y-constitutions (table 3 ).

The three populations studied as 40-male seed samples (see above) were also examined as mature plants. Nine acrocentric variants were found in the
29 males collected. Of these, five appeared identical to variants found in the seed samples while four were new types, emphasising that even 40 males is insufficient to establish the extent of within-population variation.

In the seed-sample studies, populations were very widely scattered across the country. It is thus not clear whether closely-adjacent populations show overall similarities in their Y-variation patterns. To test this four populations were examined, each about $10 \mathrm{~km}$ away from the Hendra (Cornwall) population (fig. 1; table 3 ). The Y-variation patterns in these five populations are as dissimilar as those shown by populations hundreds of kilometres distant. Clearly, then, the patterns of $\mathrm{Y}$-variation show small-scale heterogeneity.

Plants were collected from two highly toxic sites, the lead-contaminated spoil tips of Snailbeach and Darley Dale. In this extreme environment, scattered individuals of $R$. acetosa constitute the main vegetation amongst bare areas of spoil. Even under such severe conditions the amount of $\mathrm{Y}$-variation is comparable to that in the densest populations of mesic habitats (11 and 10 variants in 18 and 22 males at Darley Dale and Snailbeach respectively).

\section{Evolutionary relationships of $Y$-variants in populations}

Relationships between Y-variants cannot be ascertained because of the lack of markers along the length of these wholly heterochromatic chromo-

Table 3 The types and frequencies of Y-variants found in mature male plants from nine natural populations

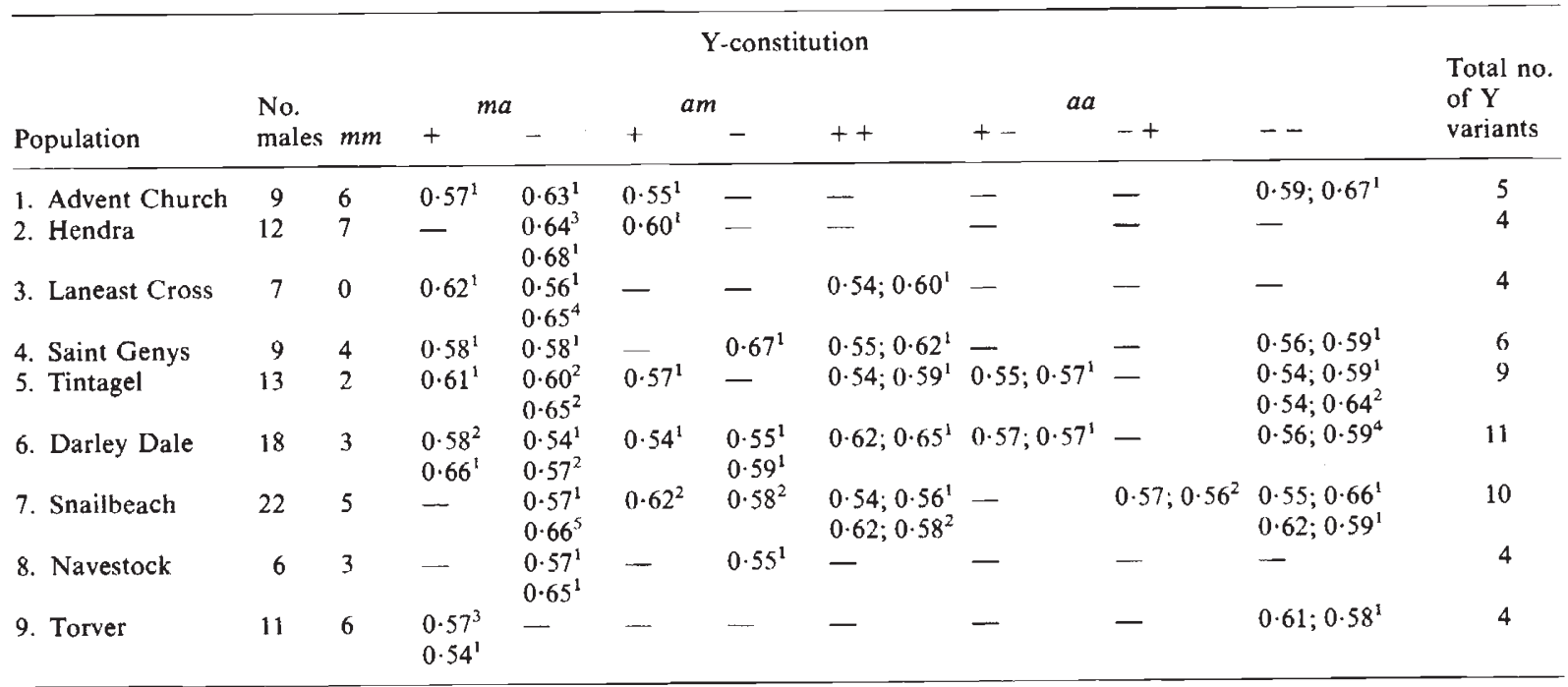


somes. In a few populations, however, comparisons of pairs of variants with one and two acrocentric chromosomes ( $a m$ or $m a$ with $a a$ ) occasionally suggest that these pairs are simply related. Clearly, these associations will most readily be noticed where extreme variants are involved. An example of this is found in the Dacre population in which an extremely acrocentric $Y_{2}$ of arm ratio 0.67 apparently occurs in combination with both a metacentric $Y_{1}\left(m a^{-}\right)$and an acrocentric of arm ratio $0.57\left(a^{-} a^{-}\right)$. Such clear examples, however, are very unusual.

One curious feature of the Y-system has been noted which suggests that there is rapid switching of the polarity of Ys. This is again most obvious in the most highly acrocentric variants. For example in the Clipston population both $\mathrm{ma}^{+}$and $m a^{-}$types have been found with the arm-ratio 0.68 (Fig. 6). Such extremely acrocentric $\mathrm{Y}_{2}$ chromosomes have only been detected twice with "+" polarity and seven times with " - " polarity. Clearly the probability of joint occurrences of these extreme variants in a single 10 -male sample is very low, less than 0.01 for this "polarity pair".

Pairs of variants which appear to differ only in polarity can be identified in 19 of the 28 small population samples (table 1). It is possible then that part of the heterogeneity of Y-chromosomes is generated by switching of the functional pairing segment from one telomere to the other without essentially disturbing the arm-ratio, effectively a "telomere-shift".

\section{Y-chromosome stability}

A common feature of heterochromatic blocks in both plants and animals is that they often show quantitative variation (Bennett, 1985). Despite the heterochromatic nature of the Ys in $R$. acetosa no quantitative variation has yet been found, suggesting that presence of a certain amount of $\mathrm{Y}$-hetero- chromatin is necessary for fertilisation to take place. In addition the partitioning of the heterochromatin between the Ys seems biologically significant since their relative sizes are remarkably constant between individuals and between populations. However, two examples of apparent $Y_{1}-Y_{2}$ interchange have been found, both interchange products retaining pairing segments. In the Dacre population, one male carried an $\mathrm{X}$-length $\mathrm{Y}$ and a small $Y$ only 60 per cent of the $X$. At meiosis the pairing pattern was undisturbed with regular trivalent formation and disjunction (Fig. 7). Pollen stainability was normal but it is not yet known whether these $\mathrm{Y}-\mathrm{Y}$ grains can effect fertilisation.

\section{DISCUSSION}

Both $\mathrm{Y}_{1}$ and $\mathrm{Y}_{2}$ chromosomes of Rumex acetosa each show continuous variation in centromere position with little indication of preferred locations within the central 40 per cent of the chromosome (Wilby and Parker, 1986). The structure of natural populations with respect to this $\mathrm{Y}$ variation has been examined here and the following points emerge: (a) all populations are highly heterogeneous with at least 4 variants in 10 randomly-sampled males; (b) any one variant is present in only a few copies per population; (c) closelyadjacent populations are as dissimilar in Y-composition as widely-separated populations; (d) most populations contain pairs of variants which differ only in the telomere at which the euchromatic pairing segment is located; (e) the number of distinct $Y$-variants is the same in small, isolated populations as in large, continuous, populations.

What factors could be involved in the maintenance of this remarkable population structure? Clearly selective or random forces are normally invoked to explain genic or chromosomal variation in natural populations (Wright, 1948). There is
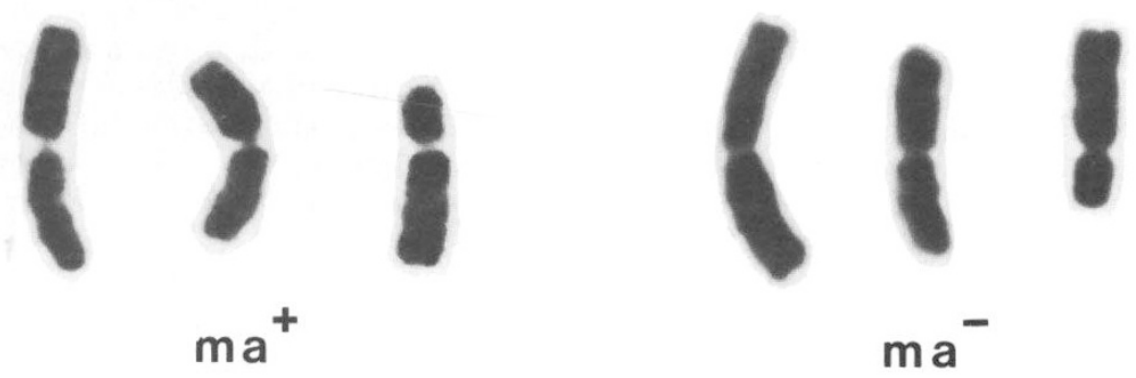

Figure 6 A pair of ma Y-variants from the Clipston population which carry extremely acrocentric $\mathrm{Y}_{2}$-chromosomes of arm-ratio 0.68 but which differ in location of the pairing segment (see text). 


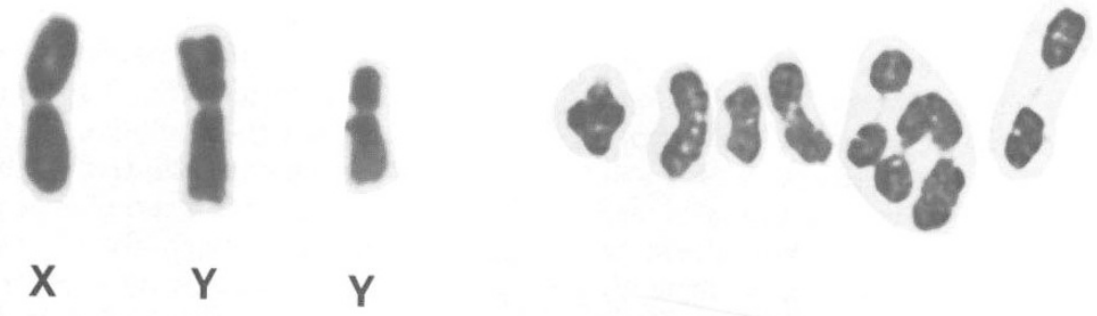

Figure 7 Possible interchange between $Y$-chromosomes. One $\mathrm{Y}$ is as large as the $\mathrm{X}$ while the other is reduced to only 60 per cent of the X-length. Sex trivalent formation and orientation is unaffected by this interchange.

some indirect evidence of selective effects on the Ys: the centromeres are never located in either distal segment and, the sizes of $\mathrm{Y}_{1}$ and $\mathrm{Y}_{2}$ are consistently maintained at 85 per cent and 75 per cent of the $X$ length. The precise location of the centromere within the central segment, however, seems neutral in its effect. Meiotic efficiency (Wilby and Parker, 1986), pollen fertility and offspring viability are unaffected by the disposition of $\mathrm{Y}$ centromeres.

If the Y-variants are effectively neutral, then population structure should simply reflect random changes in gene frequency. In particular, the small, relatively isolated populations of the highly arable areas of southern and eastern Britain would be expected to be nearly invariant with respect to the Ys. Indeed, the polymorphic autosomal variants commonly found in $R$. acetosa-supernumerary segments on chromosomes 1 and 6-show such a pattern of distribution. The Ys, by contrast, are equally variable in all populations and no populations approach fixation for Y-variants. Clearly then the effects of random changes are experienced by the autosomes but not by Ys in the same population.

An explanation of these population findings is that the site of primary constriction is relocating at a very high rate and thus in our samples any particular centric position will be detected only rarely. Rates of chromosomal rearrangement are generally considered to be of the order of $10^{-4}-10^{-5}$ per chromosome per generation (White, 1973). Specific crosses in $R$. acetos $a$ suggest that centric relocation occurs two or three orders of magnitude more frequently than expected (Wilby and Parker unpub.).

Centric shifts such as those characterising the $\mathrm{Y}$-system are usually presumed to result from pericentric inversion, a two break/two reunion event (Darlington, 1965). Other possible explanations present themselves. Recently the role of transposable elements in mutation and in the generation of rearrangements (Engels and Preston, 1984) has been much discussed. The rapid change of location of $\mathrm{Y}$-centromeres suggests that they, or some associated procentric sequence, may themselves be transposable elements, chromosome-limited like the Mp element of Zea mays (Freeling, 1984). Potential insertion sites must be restricted to the central region of the Ys.

Although some transposable elements in higher eukaryotes may play a significant role in isolation and speciation (see Kidwell et al., 1977) relatively few studies of their frequency and distribution in natural populations have been undertaken. One such study in Drosophila melanogaster shows interesting parallels to the $R$. acetosa observations. Montgomery and Langley (1983) screened the Xchromosomes of 20 males from a single wild population for the locations of three copia-like elements-copia itself, 297 and 412-each of which has multiple insertion sites. No two chromosomes in the sample had identical patterns of distribution and, interestingly, the TEs had no preferred locations, like the Y-centromeres of $R$. acetosa.

Increased mutability, perhaps associated with transposable elements, has also been implicated in the generation of $\mathrm{W}$-chromosome variants in the gekkonid lizard Gehyra purpurascens (Moritz, 1984). In this species, six variants have been found in only 30 females collected from thirteen populations. Very few females from any one population have been examined but in general populations are monomorphic and are thus quite different from those of $R$. acetosa. Further, the W-chromosome of Gehyra is euchromatic, and the evolutionary relationships of the variants can be established from C-, G- and N-banding patterns. The entirely heterochromatic Ys of $R$. acetosa give little indication of evolutionary pathways in the absence of such fine discrimination.

Evidence from a variety of organisms indicates that heterochromatin itself is highly unstable (John and Miklos, 1979). For example, Butner and Lo 
(1986) have shown that the pericentromeric heterochromatin of a mouse fusion chromosome undergoes rapid rearrangement in cultured cell lines after integration of exogenous DNA. They conclude that the fluidity of the heterochromatin is a function of the DNA environment of the cell. Thus in $R$. acetosa the centromeres of the Y-chromosomes are located in massive blocks of heterochromatin and this may itself be undergoing rapid rearrangement, leading to changes in the disposition of heterochromatin between arms. If this is the case then we might expect chromosomes in other organisms with centromeres located within heterochromatic regions to exhibit a high level of centric shifts.

A curious feature of the $\mathrm{Y}$-system is the limited range of centromere movement. This central 40 per cent of the $\mathrm{Y}$ may perhaps contain repetitious sequences with potential centromeric activity, effectively a continuous neocentromere. Single regions would be activated, perhaps determined by microtubule binding at the previous division. Binding sites may undergo random changes with a frequency of perhaps 1 in 100, giving the appearance of a shift in the position of the primary constriction without the necessity for structural rearrangement. Primary constriction sites in somatic cells are constant over time so changes may only occur during the first meiotic division which has a distinctive structural pattern of kinetochore behaviour.

Whatever the mechanism underlying the hypervariability of the $R$. acetosa Y-chromosomes it must involve a very high rate of change in some chromosomal component to give rise to this remarkable population structure.
Acknowledgements A. S. Wilby is supported by an S.E.R.C. research studentship.

\section{REFERENCES}

BENNETT, M. D. 1985. Intraspecific variation in DNA amount and the nucleotypic dimension in plant genetics. Plant Genetics, A. E. Liss Inc, pp. 283-302.

BUTNER, K. A. AND LO, C. W. 1986. High frequency DNA rearrangements associated with mouse centromeric satellite DNA. J. Mol. Biol., 187, 547-556.

DARLington, C. D. 1965. Cytology. J. and A. Churchill Ltd. London.

ENGELS, W. R. AND PRESTON, C. R. 1984. Formation of chromosome rearrangements by P-factors in Drosophila. Genetics, 107, $657-678$

FREELING, M. 1984. Plant transposable elements and insertion sequences. Ann. Rev. Plant Physiol. 35, 277-298.

JOHN, B. AND MIKLOS, G. L. G. 1979. Functional aspects of satellite DNA and heterochromatin. Int. Rev. Cytol., 58, 1-114. KIDWELL, M. G., KIDWELL, J. F. AND SVED, J. A. 1977. Hybrid dysgenesis in Drosophila melanogaster: a syndrome of aberrant traits including mutation, sterility and male recombination. Genetics, 86, 813-833.

MONTGOMERY, E. A. AND LANGLEY, C. H. 1983. Transposable elements in Mendelian populations II. Distributions of copialike elements in a natural population of Drosophila melanogaster. Genetics, 104, 473-483.

MORITZ, C. 1984. The evolution of a highly variable sexchromosome in Gehyra purpurascens (Gekkonidae). Chromosoma, 90, 111-119.

ONO, T. 1935. Chromosomen und sexualitat von Rumex acetosa. Sci. Rep. Tohuku Imp. Univ. IV, 10, 41-210.

WESTERGAARD, M. 1958. The mechanism of sex-determination in dioecious flowering plants. Adv. Gen., 9, 217-281.

WhITE, M. J. D. 1973. Animal Cytology and Evolution. C.U.P. WILBY, A. S. AND PARKER, J. S. 1986. Continuous variation in Y-chromosome structure of Rumex acetosa. Heredity, 57, 247254.

WRIGHT, S. 1948. On the roles of directed and random changes in gene frequency in the genetics of populations. Evolution, 2, 279-294. 